\title{
In vitro reconstitution of RNA primer removal in Archaea reveals the existence of two pathways
}

\author{
Ghislaine Henneke ${ }^{a, b, c, ~ * ~}$ \\ a Ifremer, UMR 6197, Laboratoire de Microbiologie des Environnements Extrêmes, BP 70, 29280 Plouzané, \\ France \\ ${ }^{\mathrm{b}}$ Université de Bretagne Occidentale, UMR 6197, Laboratoire de Microbiologie des Environnements Extrêmes, \\ 29280 Plouzané, France \\ ${ }^{c}$ Centre National de la Recherche Scientifique, UMR 6197, Laboratoire de Microbiologie des Environnements
} Extrêmes, 29280 Plouzané, France

*: Corresponding author : Ghislaine Henneke, email address : ghenneke@ifremer.fr

\begin{abstract}
:
Using model DNA substrates and purified recombinant proteins from Pyrococcus abyssi, I have reconstituted the enzymatic reactions involved in RNA primer elimination in vitro. In my dual-labelled system, polymerase D performed efficient strand displacement DNA synthesis, generating 5'-RNA flaps which were subsequently released by Fen1, before ligation by Lig1. In this pathway, the initial cleavage event by RNase HII facilitated RNA primer removal of Okazaki fragments. In addition, I have shown that polymerase $B$ was able to displace downstream DNA strands with a single ribonucleotide at the $5^{\prime}$-end, a product resulting from a single cut in the RNA initiator by RNase HII. After RNA elimination, the combined activities of strand displacement DNA synthesis by polymerase B and flap cleavage by Fen 1 provided a nicked substrate for ligation by Lig1. The unique specificities of Okazaki fragment maturation enzymes and replicative DNA polymerases strongly support the existence of two pathways in the resolution of RNA fragments.
\end{abstract}

Keywords: Archaea ; DNA ligase ; DNA polymerase ; nuclease ; Okazaki fragment

Abbreviations used: Cy5, indodicarbocyanine ; FAM, 6-carboxyfluorescein ; Fen1, flap endonuclease 1 ; Lig1, DNA ligase 1 ; Pab, P. abyssi ; PCNA, proliferating-cell nuclear antigen ; PIP, PCNAinteracting protein ; pol $\alpha$, polymerase $\alpha$; pol $B$, family $B$ DNA polymerase ; pol $D$, family D DNA polymerase ; pol $\delta$, polymerase $\delta$. 


\section{INTRODUCTION}

Accurate elimination of RNA initiators at the replication fork is a crucial process to ensure genome integrity. In eukaryotic cells, RNA initiators, 7-12 nucleotides (nt) in length, are generated by the primase activity of DNA polymerase $\alpha /$ primase (pol $\alpha /$ primase) and prime DNA synthesis of both leading and lagging strands. These initiating RNA primers are then further extended to around 20 deoxynucleotides by the polymerase activity of pol $\alpha /$ primase before handing over to the replicative DNA polymerase [1]. Whereas the leading strand is continuously replicated by DNA polymerase $\varepsilon$ (pol $\varepsilon$ ) [2], the lagging strand of opposite polarity is discontinuously synthesized by DNA polymerase $\delta$ (pol $\delta$ ) [3] and consists of multiple segments called Okazaki fragments, each of roughly 130-150 nt [4]. There is considerable evidence that pol $\delta$ displaces the RNA initiator of the downstream Okazaki fragment and creates 5'-tails [5]. Short tail intermediates are cleaved by the flap endonuclease (Fen1) to generate a nick for ligation by DNA ligase 1 (Lig 1), a prevailing pathway in wildtype replicating cells supported by genetic and biochemical evidence [6-8]. Failure of this process occasionally leads to long flap formation [6], a situation that requires the coordinated activities of two nucleases, Dna2 and Fen1, and the single-stranded binding protein, replication protein A (RPA), to fully ensure RNA primer removal in yeast [9-12]. Human Dna2 has recently been described as a nuclease for processing mitochondrial Okazaki fragments [13]. Ultimately, the dysfunctional maturation mechanism can be counteracted by an alternative pathway involving two nucleases, RNase $\mathrm{H}$ and Fen1 [14]. In the second pathway preliminary removal of RNA initiators is conducted by RNase $\mathrm{H}$, leaving a single ribonucleotide at the 5'-end of the downstream primer, before displacement DNA synthesis by pol $\delta$ holoenzyme. The resulting flap is then cleaved by Fen1, generating a nick for DNA ligation [15]. While Okazaki fragment processing has been intensively examined, RNA primer removal at the leading strand remains unknown in eukaryotic cells.

In archaeal cells, RNA-primed replication intermediates, similar to those formed during eukaryotic DNA replication, have been observed in euryarchaea and crenarchaea [16], arguing for either RNA or RNA-DNA primers at the archaeal replication fork. RNA priming seems to involve a eukaryotic-like primase complex [17-21]. However, there is currently no direct evidence that archaeal primases are responsible for RNA primer elongation. Biochemical data accumulated so far indicated that the family D DNA polymerase (pol D) would extend RNA primers both at the leading and lagging strands in euryarchaea, followed by DNA synthesis at the lagging strand [22]. Furthermore, switching from pol D to the replicative family B DNA polymerase (pol B) on the homotrimeric DNA sliding clamp (PCNA) has been shown to confer processive DNA synthesis at the leading strand [23]. As in bacteria and eukaryotes, the DNA sliding clamp is an essential protein [24-26], acting as a stimulatory factor to pol B and pol D in archaeal DNA transactions [27-29]. In crenarchaea, multiple family B DNA polymerases (designated B1, B2 and B3) [30, 31] have been identified and observed mediating interactions with their cognate PCNA [32, 33]. Crenarchaeal PCNA organizes as a heterotrimer and contacts simultaneously DNA polymerase B1, Lig1, or Fen1, imposing a defined architecture at the lagging strand [33, 34]. More recently functional assessment of this structural organization argues for the coordination of multiple enzyme activities by a single PCNA in Okazaki fragment maturation [35]. On the other hand, euryarchaeal PCNA interacts with maturation enzymes [25, 36, 37], raising the possibility that a eukaryotic-type Okazaki fragment maturation process is functional in these cells. However, the molecular mechanism required for RNA primer elimination at the leading strand remains to be determined in archaea.

Pyrococcus abyssi (P. abyssi), a hyperthermophilic and anaerobic euryarchaeon that grows optimally at $95^{\circ} \mathrm{C}$ and $\mathrm{pH} 6.8$ [38], has proved to be an excellent model to analyze DNA 
replication because it contains a simplified eukaryotic-like replication machinery [39]. Consistent with this statement, the structure and size of RNA-primed replication intermediates ( $\sim 150$ nt in length with RNA primer of $\sim 12 \mathrm{nt}$ ) are similar to eukaryotic Okazaki fragments [16]. P. abyssi possesses a core version of the eukaryotic maturation enzymes: two DNA polymerases (pol B and pol D), Fen1, Lig1, PCNA and a type 2 RNase H (RNase HII). In order to examine the specific reactions involved in RNA primer elimination, these recombinant proteins have been purified and used to reconstitute Okazaki fragment processing in vitro. We found that two functional pathways may be responsible for RNA primer removal in $P$. abyssi. 


\section{EXPERIMENTAL}

Enzymes and Proteins. All the enzymes used were prepared and purified as previously described [25, 40, 41] with the exception of Lig1 which was purified with Ni-NTA spin columns (Qiagen). The purity of proteins was controlled by SDS-PAGE (data not shown).

DNA substrates. Oligonucleotides were chemically synthesized and HPLC-purified by Eurogentec (Belgium), with all sequences listed in Supplementary Table 1. The location of the 5'-FAM or 3'-Cy5 fluorescent-label on primer is differentiated by a full circle or an asterisk, respectively. Construction of the primer-template minicircle was previously described [42]. To generate the substrates, complementary primers were annealed to the respective template as previously described [22, 42] and the resulting substrates were gelpurified from excess primer. The dual-labeled substrate allows simultaneous detection of upstream and downstream primers in a single reaction, providing multiple correlated data from a single reaction.

DNA replication assays. PabpolB assays $(10 \mu \mathrm{l})$ contained $50 \mathrm{mM}$ Tris- $\mathrm{HCl}(\mathrm{pH} 8.8), 50$ $\mathrm{mM} \mathrm{KCl}, 1 \mathrm{mM}$ dithiothreitol, $2 \mathrm{mM} \mathrm{MgCl}_{2}, 0.25$ pmol (3'-OH ends) of fluorescent-labeled primed synthetic substrate and $200 \mu \mathrm{M}$ of each dNTPs (additional components are mentioned in Figure legends). Various amounts of enzymes and proteins were incubated with appropriate substrate as indicated in Figure legends. Reactions were performed at $60^{\circ} \mathrm{C}$ for $30 \mathrm{~min}$, unless otherwise stated. Reactions were terminated by addition of $10 \mu \mathrm{l}$ of formamide containing 10 $\mathrm{mM}$ EDTA, followed by heating at $95^{\circ} \mathrm{C}$ for $5 \mathrm{~min}$. Labeled products were separated by denaturing PAGE as already described [42]. Products resulting from coupled-activity of PabpolB and PabRNase HII were resolved on $8 \%$ native PAGE, followed by staining with SYBR Gold (Invitrogen). Product analyses were performed with an Imager Typhoon 9400 (GE Healthcare) and quantification was carried out using ImageQuant 5.2 software. In all cases, the percentage of substrate hydrolysis, displaced strands or ligation was determined by the products / (products + substrate) ratio. PabpolD assays $(10 \mu \mathrm{l})$ contained $50 \mathrm{mM}$ Tris-HCl

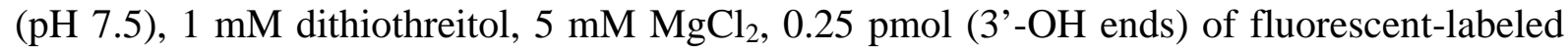
primed synthetic oligonucleotide and $200 \mu \mathrm{M}$ of each dNTPs (unless otherwise stated). Reactions were performed and analyzed as described above. 


\section{RESULTS}

\section{PabRNase HII is required to initiate strand displacement DNA synthesis by PabpolB, followed by PabFen1 cleavage}

We initially determined whether active ribonuclease function could be required to provide an appropriate substrate for PabpolB strand displacement as recently suggested [43]. To address this question, we used a minicircle DNA template annealed to an unlabeled RNA-DNA primer, since PabRNase HII cleavage would have hampered the detection of PabpolB displacement products. Our detection assay was based on fluorescent gel staining which allowed detection of the products from the combined enzymatic activities. As expected, PabRNase HII alone displayed efficient cleavage activity (Figure 1A, lane 3). In this case, the bands representing cleavage products shifted to lower positions (Figure 1A, compare lane 1 and lane 3). When PabpolB was titrated into the reaction, a distribution of longer products appeared (Figure 1A, lanes 4-7). Strand displacement mostly occurred on substrates in which RNA initiators, annealed to the minicircle DNA template, have been hydrolyzed, as shown by the decrease of short products. PabRNase HII specifically cuts one nucleotide upstream of the RNA-DNA junction, leaving a single ribonucleotide at the 5'-end of the primer on this labeled substrate (Supplementary Figure S1A). Control reactions were carried out with PabpolB alone and the results were consistent with its inability to displace RNA-DNA segments (Figure 1A, lane 2) (see also Supplementary Figure S2C). Moreover, addition of increasing amounts of PabPCNA did not initiate strand displacement activity by PabpolB (Supplementary Figure S2D). These results suggested that initial nuclease activity on RNA initiators by PabRNase HII is required to promote displacement of the resulting 5'phosphorylated junction ribonucleotide attached to DNA by PabpolB. It is of interest to note that strand displacement activity by PabpolB was not dependent of the nature of the monoribonucleotide (data not shown). In a plausible model for RNA primer elimination, one could envisage that after strand invasion by the polymerase, the resulting flap could be cut by the 5'-flap endonuclease, PabFen1. This prediction was assessed on the minicircle DNA template annealed to a fluorescent 5'-end labeled monoribonucleotide DNA primer (Figure 1B). This substrate allows a direct measurement of strand displacement DNA synthesis coupled to flap cleavage. In the conditions tested, PabpolB alone carried out strand displacement DNA synthesis with accumulated products of about $97 \mathrm{nt}$ in length ( $16 \%$ displ after $10 \mathrm{~min}$ ), resulting from $\sim 10 \mathrm{nt}$ of strand displacement (Figure 1B, lane 2). When PabFen1 was titrated into the reaction, the major distribution of cleavage products was in the 3-6 nt size range with a greater accumulation of shorter fragments at the highest enzyme concentration (Figure 1B, lane 7) ( 15 \% cleavage). Control assays containing PabFen1 without DNA polymerase showed no cleavage products (Figure 1B, lane 8). Interestingly, a direct relationship between the loss of 5'-flaps and the formation of Fen 1 cleavage products was noticeable. These results indicate that the coordination between PabpolB and PabFen1 is functional and preferentially leads to cleavage of short flaps. This was not the consequence of a limited size flap cleavage capability by PabFen 1 since the enzyme could catalyze the hydrolysis of 16 nt flaps (Supplementary Figure S1B). When PabFen1 was supplemented at different time intervals, the distribution of cleavage products varied (Figure 1C). At time 0, 60 $\%$ of cleavage products were in the 1-12 nt size range. At time $5 \mathrm{~min}$, a steady state situation between shorter and longer products was detected. At time $15 \mathrm{~min}, 62 \%$ of cleavage products were 20-25 nt in size. Similar to what was observed when PabFen1 was present from the beginning of the reaction, shorter products (1-12 nt) were preferentially generated. However, when PabpolB was allowed to displace longer DNA tracts (at least $25 \mathrm{nt}$ in $15 \mathrm{~min}$ ) prior to the addition of PabFen1 longer cleavage products were formed. Thus, these results show that the flap continuously generated by PabpolB strand displacement can be cut by PabFen1. 


\section{Coordinated activities of PabpolB and PabFen1 on monoribonucleotide-primed DNA replication intermediates produce ligatable nicks}

To reconstitute the final step of RNA primer removal, a dual-labeled system was used to allow simultaneous detection of the resulting upstream and downstream products in a single reaction. The substrate mimics an RNA-primed replication intermediate which can be generated during the maturation process. On this substrate, complete strand displacement DNA synthesis by PabpolB ( 66 \% displ) was observed, as shown by the synthesis of fulllength products (87 nt) (Figure 2, lane 5; see FAM panel). Strand displacement DNA synthesis of the monoribonucleotide DNA hybrid was attributed to pausing of the polymerase in the vicinity of the $57 \mathrm{nt}$ position. Two pause sites at $57 \mathrm{nt}$ and $62 \mathrm{nt}$, corresponding to the "nick" position (57 nt) and +5 position (62 nt), were also observed (Figure 2, lane 5). Furthermore, a faint band appeared at a lower size (80 nt) than the full-length products (87 nt), indicating aborted DNA synthesis. This is indicative of the presence of persistent secondary structures formed by the DNA, as deduced by the primary sequence, presumably causing the dissociation of the polymerase from the DNA template. Addition of PabFen1 did not alter the efficiency of PabpolB strand displacement DNA synthesis ( $69 \%$ displ), (Figure 2, lane 6; see FAM panel) nor the presence of pause sites. PabFen1 cleavage activity mainly released products 3-12 nt in length (Figure 2, lane 6; see Cy5 panel). These results are in agreement with those obtained in Figure 1B showing that PabFen1 cleaves the flap continuously generated by PabpolB strand displacement, releasing products of a preferential size range.

PabPCNA has been described as a molecular platform since it specifically interacts not only with DNA polymerases but also with maturation enzymes [25, 27]. When added to PabpolB in the maturation assay, strand displacement DNA synthesis was not affected ( $71 \%$ displ) (Figure 2, lane 7; see FAM panel), although stimulation of primer extension was observed with the control (Supplementary Figure S2D). On the other hand, PabPCNA helped to bypass secondary structures (62 and $80 \mathrm{nt}$ ), resulting in a higher amount of full-length products (87 nt) (Figure 2, compare lanes 5 and 7; see FAM panel). In the presence of PabFen1 and PabPCNA, cleavage efficiency remains constant ( 42 \%) but shorter cleavage products were released by PabFen1 (3-15 nt) (Figure 2, compare lanes 6 and 8 in Cy5 panel).

When PabLig1 was included in the strand displacement reaction, a dual-labeled band migrating at a higher position than the full-length synthesis product (87 nt) was observed (Figure 2, lane 15; see FAM and Cy5 panels). This DNA fragment, running slower than the full-length extension product because of the attached Cy5, represented ligation products. In these conditions, the total percentage of ligated products was $61 \%$. Ligation obviously resulted from a combination of PabpolB strand displacement and PabFen1 cleavage, as deduced by the loss of PabFen1 cleavage products (Figure 2, lane 6 compared with lane 15 in Cy5 panel). Moreover, PabpolB strand displacement DNA synthesis was strongly stimulated by PabLig1 ( 91 \% displ), as observed by the accumulation of full-length products and the concomitant disappearance of pause sites (Figure 2, compare lanes 5 and 14; see FAM panel). This unanticipated result suggests that the polymerase and DNA ligase likely cooperate to enhance strand displacement. Addition of PabPCNA did not impact on ligation efficiency (Figure 2, compare lanes 15 and 17 in Cy5 panel). This observation is interesting, since functional interaction between PabPCNA and PabLig1 was detectable (Supplementary Figure S4B). Altogether, these data demonstrate the coordination of strand displacement and flap cleavage activities by PabpolB and PabFen1 to achieve efficient DNA ligation by PabLig1 on DNA replication intermediates. 


\section{Coordinated activities of PabpolD and PabFen1 on RNA-primed DNA replication intermediates produce ligatable nicks}

Previous reports indicated that PabpolD is the lagging strand DNA polymerase, performing RNA primer extension and strand displacement in the presence of PabPCNA [22, 23]. Others demonstrated that Okazaki fragments are initiated with short RNA primers ( $12 \mathrm{nt})$ followed by extension with DNA [16]. To verify whether PabpolD was capable of displacing the RNA initiator at the 5'-end of the downstream primer, our reconstituted dual-labeled system was used. Time course experiments indicated that PabpolD displaced RNA-DNA primers (Supplementary Figure S2A), this activity was further stimulated by PabPCNA (Supplementary Figure S2B). However, when these reactions were performed with PabpolB alone (Supplementary Figure S2C) or in the presence of PabPCNA (Supplementary Figure S2D), strand displacement DNA synthesis never occurred. The incubation of PabFen1 and PabpolD in the maturation assay yielded extension products up to 87 nt (Figure 3, lane 6; see FAM panel) and cleaved flaps 2-12 nt in length (see Cy5 panel). Cleavage products accumulated (12\%) to the same extent than strand displacement products (17\% displ) generated by PabpolD. Primer extension and DNA synthesis through pause sites in the vicinity of the $57 \mathrm{nt}$ (nick) position were moderately enhanced in the presence of PabFen1 (Figure 3, compare lanes 5 and 6; see FAM panel). When PabPCNA was added to the same reactions, strand displacement DNA synthesis (70 \% displ) and PabFen1 cleavage activity (42 $\%$ ) were markedly increased, but the pattern of cleavage products was not affected (Figure 3, lane 6 compared with lane 8 in FAM and Cy5 panels). Thus, our results show that PabFen1 cuts the flaps (not exceeding 12 nt in length) that are continuously generated by PabpolD. In order to reconstitute the final step of Okazaki fragment maturation, DNA ligase 1 was added. As expected, ligation only occurred in the presence of PabFen1 (Figure 3, lane 15; see Cy5 panel). All ligated products derived from the combination of flap creation and total elimination of the RNA initiator. Consistently, control experiments indicated that PabLig1 can not catalyze the formation of the phosphodiester bond between DNA and RNA (Supplementary Figure 4C). On the other hand, DNA ligation proceeded with $29 \%$ efficiency when the monoribonucleotide downstream primer was used (Supplementary Figure 3, lane 15; see Cy5 panel). This suggests that elimination of the RNA portion of the downstream primer, prior to strand displacement DNA synthesis, likely contributes to enhance the maturation reaction. Although unexpected, addition of DNA ligase 1 clearly stimulated strand displacement DNA synthesis by PabpolD (6-fold) (Figure 3, lane 14 compared with lane 5 in FAM panel). Moreover, addition of PabPCNA resulted in an increase of strand displacement products (70 \% displ) and PabFen1 cleavage efficiency (40\%), while not affecting DNA ligation (Figure 3, lane 17 in both panels). Altogether, our data demonstrate that sequential enzymatic reactions by PabpolD, PabFen1 and PabLig1 lead to the generation of displaced strands which can be processed into ligatable products to create the continuous doublestranded DNA.

\section{Initial cleavage event by PabRNase HII facilitates RNA primer removal of Okazaki fragments}

Although our findings showed that PabFen1 provides the nuclease activity for removal of RNA initiators on Okazaki fragments, a role for PabRNase HII could not be totally excluded. Our recent results have shown that PabRNase HII made structure-specific endonucleolytic cleavage in the RNA initiator, leaving a single ribonucleotide at the 5'-end of the RNA-DNA junction [43] (see also Supplementary Figure 1A), an activity which can occur once at the leading strand and much more frequently at the lagging strand. Therefore, we assessed the ability of the nuclease to degrade the RNA initiator using the reconstituted assay in the presence of all the maturation enzymes and either PabpolD or PabpolB. PabRNase HII could 
hydrolyze the substrate generating a primary product, exactly the length (19 nt) of the DNA portion with a single ribonucleotide remaining at the 5'-end (Figure 4, lanes 2 and 11 in Cy5 panel). Neither specificity nor efficiency of cleavage by PabRNase HII was modified upon different combinations of maturation enzymes and replicative DNA polymerases (Figure 4, lanes 3-7 and lanes 12-16; see Cy5 panel). However, PabpolD strand displacement DNA synthesis was stimulated upon addition of PabRNase HII (Figure 4, compare lanes 8 and 9; FAM panel), as shown by the absence of pause products in the vicinity of the downstream primer (57 nt in length) and the increase of intensity of the full-length extension products (up to $81 \%$ displ). Interestingly, the amount of ligation products, which resulted from total RNA elimination, increased by approximately three times in the presence of PabRNase HII (Figure 4, compare lanes 8 and 9; Cy5 panel).

Consistent with the results shown in Figure 1A, PabRNase HII was initially required to provide the monoribonucleotide substrate intermediate, thus promoting strand displacement DNA synthesis by PabpolB (Figure 4, lane 16; FAM panel). Indeed, all extension products accumulated at the position of the nick (57 nt) when PabRNase HII was omitted from the maturation assay (Figure 4, lane 17; FAM panel), and none of the maturation enzymes tested were able to initiate strand displacement activity. These results confirm the inability of PabpolB to displace RNA primers as shown in Supplementary Figure S2C-D. Accordingly, maturation assays performed initially with PabRNase HII followed by the addition of all maturation enzymes and PabpolB resulted in the generation of ligation products (Figure 4, lane 18; Cy5 panel). Altogether, these results show that the reconstitution of RNA primer excision with initial RNase HII cleavage event is functional with both replicative DNA polymerases, and that it implies timely orchestrated enzymatic reactions. 


\section{DISCUSSION}

In this report, we have successfully reconstituted the reactions of RNA primer removal in vitro by using purified enzymes from the hyperthermophilic euryarchaeon Pyrococcus abyssi. We reported that pol B strand displacement DNA synthesis was restricted to a single ribonucleotide attached to a DNA segment. In this pathway, the initial cleavage event by RNase HII was shown to be required to provide a suitable substrate for pol B strand displacement. These data indicate that the combined activities of pol B and RNase HII are involved in the elimination of the RNA initiator. Moreover, they are in agreement with the recent findings that assigned pol $\mathrm{B}$ a role in leading strand DNA synthesis $[22,23]$ and described RNase HII as a potent maturation enzyme [43]. As a result of the action of the two enzymatic activities, flap structural intermediates were recognized and released by Fen1. Flap removal was entirely dependent on the tight coordination of strand displacement DNA synthesis and flap cleavage. In this mechanism, Fen1 cuts the flaps that are continuously generated by pol B, releasing products $2-12 \mathrm{nt}$ in length. However, it is plausible that a delay in Fen1 action (shown in this paper) or uncontrolled strand displacement as observed in eukaryotes [44], results in the formation of lengthy flap structures. Although the occurrence of this event awaits further in vivo assessment in archaea, the resolution of these structural intermediates might involve a specific molecular mechanism yet undiscovered. Eventually, DNA ligase 1 was recruited to seal the resulting DNA nick. Unexpectedly, Lig1 was found to stimulate strand displacement DNA synthesis by PabpolB. Time course experiments indicated that Lig1 accelerates the rate of Fen1 cleavage activity, thus facilitating the displacement of the 5'-end monoribonucleotide primer by pol B (data not shown). Therefore, Lig1 seems to be an important component of maturation processing in archaea.

On model Okazaki fragment substrates [16], pol D exclusively carried out RNA strand displacement DNA synthesis in the presence of PCNA, acting as a stimulatory clamp for strand displacement and primer utilization. The dynamic process of strand displacement DNA synthesis by pol D coupled with Fen1 cleavage was clearly identified. These combined activities gave rise to a preferential size distribution of released flaps, with flaps not exceeding $12 \mathrm{nt}$ in length. Consistent with the total elimination of the RNA portion of Okazaki fragments, ligation only occurred on substrates containing DNA ends with a 3'-OH termini and a 5'-phosphate. Moreover, we observed that RNase HII could participate early in this pathway, cutting specifically at the phosphodiester bond of the nucleotide 5' of the RNADNA junction. Removal of the last 5'-ribonucleotide was then performed by the action of pol $\mathrm{D}$ and Fen1 prior to DNA ligation. Although unanticipated, we found that Lig1 and RNase HII stimulated strand displacement activity by pol D, and that Fen1 slightly enhanced primer utilization. Such functional interactions have already been described in eukaryotes [45]. Further investigation is now required to analyze the specificity of the interplay between these enzymatic activities. This would contribute, for instance, to explain the weak ligation efficiency observed with all maturation enzymes on RNA-primed DNA replication intermediates.

Based on these findings, a model for RNA primer removal during DNA replication is represented in Figure 5. In the RNase HII-pathway, RNase HII initially degrades the RNA initiator. Following primer extension, either pol B or pol D encounters the double-stranded region, displacing the monoribonucleotide primer. The resulting flap is then recognized and cleaved by Fen1. As a final step, the resulting DNA nick is sealed by Lig1 to yield the continuous double-stranded DNA. In the absence of RNase HII, pol D encounters and then displaces the downstream RNA-DNA primer. Elimination of RNA flaps relies on the combined activities of pol D strand displacement DNA synthesis and Fen1 cleavage. As a final step, a DNA nick is created and subsequently sealed by Lig1. 
Unlike the heterotrimeric crenarchaeal PCNA which has been recently shown to coordinate multiple enzymatic activities in the removal of RNA primers on a single ring [35], the homotrimeric euryarchaeal PCNA seems to have evolved a distinct mechanism. Through this study, we clearly demonstrated that PCNA could contribute to further enhance DNA replication through double-stranded DNA structures rather than stimulation of primer utilization by pol B, while stimulating both properties by pol D. Interestingly, a previous report identified one and two functional PCNA binding motifs in pol B and pol D, respectively [27]. PCNA binding was different for the two DNA polymerases, and it was suggested that the mechanism involved in polymerase recruitment and stimulation at the replication fork might be different. Our maturation assay gave the first evidence that PCNA can indeed confer differential properties to the DNA synthesis activities of the replicative DNA polymerases. On the other hand, the role of PCNA as a processivity factor helping the polymerase to disrupt hydrogen bonding of the downstream primer during strand displacement DNA synthesis was preserved [22]. When all enzymes were combined, PCNA exerted a stimulatory effect on Fen1 cleavage but did not influence DNA ligation by Lig1. It is probable that PCNA interaction with DNA polymerases and Fen1 might sterically prevent the simultaneous binding of additional partners. Similarly, it has been shown that the eukaryotic DNA ligase 1 do not function with the highest efficiency in the presence of the homotrimeric PCNA. It was proposed that Fen 1 and/or the replicative DNA polymerase $\delta$ likely competed with DNA ligase 1 for the same binding site(s) on PCNA [6]. Therefore, it appears that the presence of three identical surfaces on PCNA may restrict and direct the access of target proteins. In this respect, Creze and coworkers recently described the functional and structural characterization of the complex formed between PCNA and NucS, a structure-specific DNA endonuclease. They demonstrated that only one molecule of the NucS homodimer binds to the outside surface of the PCNA homotrimer via the PIP/IDCL interface, leading to the conclusion that this particular stoichiometry could be due to long range conformational changes on PCNA that in turn could regulate the recruitment of additional partners [46]. Other factor(s) (e.g., local protein concentrations and/or affinities of the PIP motifs of the various proteins) might also be required for in vivo coordination of these functional interactions during the processing maturation in euryarchaea. It is interesting to note that post-translational modifications of proteins involved in DNA transactions have, as yet, not been identified in archaea.

Our in vitro reconstitution assay has provided mechanistic insights into how proteins known to be essential in archaea [24, 25] ensure the accurate and timely processing of RNA primers, an activity which can occur once at the leading strand or much more frequently at the lagging strand. In agreement with the recent study of Beattie and Bell [35], it appears that archaea evolved a minimal 5'-RNA containing tail as a safety control. In this mechanism, Fen1 cleaves the tails as soon as they are produced by the replicative DNA polymerases. However, it remains possible that other factors may be involved in this pathway. RNase HII is one of the plausible candidates which could participate in RNA primer removal early in the maturation reaction, before displacement by replicative DNA polymerases. Intriguingly, these findings resemble predicted mechanistic pathways of RNA primer processing in mitochondria [47]. Thus, euryarchaea could serve as a good model to understand these highly ordered processes occurring not only in the nucleus of eukaryotes but also in mitochondria. 


\section{ACKNOWLEDGEMENTS}

I am grateful to Stéphane Koundrioukoff, Didier Flament and Tom Killelea for critical reading of the manuscript. I thank Hannu Myllykallio for providing the expression clones encoding PabRNase HII, PabLig1 and PabFen1.

\section{FUNDING}

This work was supported by the French institute of marine research and exploitation (Ifremer) and Ifremer EDROME (Carnot Institute). G.H. was supported by grant ANR-10-JCJC-150101 from the National Research Agency. 


\section{REFERENCES}

1 Waga, S. and Stillman, B. (1994) Anatomy of a DNA replication fork revealed by reconstitution of SV40 DNA replication in vitro. Nature. 369, 207-212

2 Pursell, Z. F., Isoz, I., Lundstrom, E. B., Johansson, E. and Kunkel, T. A. (2007) Yeast DNA polymerase epsilon participates in leading-strand DNA replication. Science. 317, 127130

3 Nick McElhinny, S. A., Gordenin, D. A., Stith, C. M., Burgers, P. M. and Kunkel, T. A. (2008) Division of labor at the eukaryotic replication fork. Mol. Cell. 30, 137-144

4 Anderson, S. and DePamphilis, M. L. (1979) Metabolism of Okazaki fragments during simian virus 40 DNA replication. J. Biol. Chem. 254, 11495-11504

5 Rossi, M. L. and Bambara, R. A. (2006) Reconstituted Okazaki fragment processing indicates two pathways of primer removal. J. Biol. Chem. 281, 26051-26061

6 Ayyagari, R., Gomes, X. V., Gordenin, D. A. and Burgers, P. M. (2003) Okazaki fragment maturation in yeast. I. Distribution of functions between FEN1 AND DNA2. J. Biol. Chem. 278, 1618-1625

7 Maga, G., Villani, G., Tillement, V., Stucki, M., Locatelli, G. A., Frouin, I., Spadari, S. and Hubscher, U. (2001) Okazaki fragment processing: modulation of the strand displacement activity of DNA polymerase delta by the concerted action of replication protein A, proliferating cell nuclear antigen, and flap endonuclease-1. Proc. Natl. Acad. Sci. U.S.A. 98, 14298-14303

8 Tishkoff, D. X., Filosi, N., Gaida, G. M. and Kolodner, R. D. (1997) A novel mutation avoidance mechanism dependent on $S$. cerevisiae RAD27 is distinct from DNA mismatch repair. Cell. 88, 253-263

9 Bae, S. H., Bae, K. H., Kim, J. A. and Seo, Y. S. (2001) RPA governs endonuclease switching during processing of Okazaki fragments in eukaryotes. Nature. 412, 456-461

10 Bae, S. H. and Seo, Y. S. (2000) Characterization of the enzymatic properties of the yeast dna2 Helicase/endonuclease suggests a new model for Okazaki fragment processing. J. Biol. Chem. 275, 38022-38031

11 Budd, M. E. and Campbell, J. L. (1997) A yeast replicative helicase, Dna2 helicase, interacts with yeast FEN-1 nuclease in carrying out its essential function. Mol. Cell. Biol. 17, 2136-2142

12 Kang, H. Y., Choi, E., Bae, S. H., Lee, K. H., Gim, B. S., Kim, H. D., Park, C., MacNeill, S. A. and Seo, Y. S. (2000) Genetic analyses of Schizosaccharomyces pombe dna2(+) reveal that dna2 plays an essential role in Okazaki fragment metabolism. Genetics. 155, 1055-1067

13 Zheng, L., Zhou, M., Guo, Z., Lu, H., Qian, L., Dai, H., Qiu, J., Yakubovskaya, E., Bogenhagen, D. F., Demple, B. and Shen, B. (2008) Human DNA2 is a mitochondrial nuclease/helicase for efficient processing of DNA replication and repair intermediates. Mol. Cell. 32, 325-336

14 Qiu, J., Qian, Y., Frank, P., Wintersberger, U. and Shen, B. (1999) Saccharomyces cerevisiae RNase $\mathrm{H}(35)$ functions in RNA primer removal during lagging-strand DNA synthesis, most efficiently in cooperation with Rad27 nuclease. Mol. Cell. Biol. 19, 83618371

15 Kao, H. I. and Bambara, R. A. (2003) The protein components and mechanism of eukaryotic Okazaki fragment maturation. Crit. Rev. Biochem. Mol. Biol. 38, 433-452

16 Matsunaga, F., Norais, C., Forterre, P. and Myllykallio, H. (2003) Identification of short 'eukaryotic' Okazaki fragments synthesized from a prokaryotic replication origin. EMBO Rep. 4, 154-158 
17 Desogus, G., Onesti, S., Brick, P., Rossi, M. and Pisani, F. M. (1999) Identification and characterization of a DNA primase from the hyperthermophilic archaeon Methanococcus jannaschii. Nucleic Acids Res. 27, 4444-4450

18 Lao-Sirieix, S. H. and Bell, S. D. (2004) The heterodimeric primase of the hyperthermophilic archaeon Sulfolobus solfataricus possesses DNA and RNA primase, polymerase and 3'-terminal nucleotidyl transferase activities. J. Mol. Biol. 344, 1251-1263

19 Le Breton, M., Henneke, G., Norais, C., Flament, D., Myllykallio, H., Querellou, J. and Raffin, J. P. (2007) The heterodimeric primase from the euryarchaeon Pyrococcus abyssi: a multifunctional enzyme for initiation and repair? J. Mol. Biol. 374, 1172-1185

20 Liu, L., Komori, K., Ishino, S., Bocquier, A. A., Cann, I. K., Kohda, D. and Ishino, Y. (2001) The archaeal DNA primase: biochemical characterization of the p41-p46 complex from Pyrococcus furiosus. J. Biol. Chem. 276, 45484-45490

21 Matsui, E., Nishio, M., Yokoyama, H., Harata, K., Darnis, S. and Matsui, I. (2003) Distinct domain functions regulating de novo DNA synthesis of thermostable DNA primase from hyperthermophile Pyrococcus horikoshii. Biochemistry. 42, 14968-14976

22 Henneke, G., Flament, D., Hubscher, U., Querellou, J. and Raffin, J. P. (2005) The hyperthermophilic euryarchaeota Pyrococcus abyssi likely requires the two DNA polymerases D and B for DNA replication. J. Mol. Biol. 350, 53-64

23 Rouillon, C., Henneke, G., Flament, D., Querellou, J. and Raffin, J. P. (2007) DNA Polymerase Switching on Homotrimeric PCNA at the Replication Fork of the Euryarchaea Pyrococcus abyssi. J. Mol. Biol. 369, 343-355

24 Berquist, B. R., DasSarma, P. and DasSarma, S. (2007) Essential and non-essential DNA replication genes in the model halophilic Archaeon, Halobacterium sp. NRC-1. BMC Genet. 8, 31

25 Meslet-Cladiere, L., Norais, C., Kuhn, J., Briffotaux, J., Sloostra, J. W., Ferrari, E., Hubscher, U., Flament, D. and Myllykallio, H. (2007) A novel proteomic approach identifies new interaction partners for proliferating cell nuclear antigen. J. Mol. Biol. 372, 1137-1148

26 Zhang, C., Guo, L., Deng, L., Wu, Y., Liang, Y., Huang, L. and She, Q. (2010) Revealing the essentiality of multiple archaeal pcna genes using a mutant propagation assay based on an improved knockout method. Microbiology. 156, 3386-3397

27 Castrec, B., Rouillon, C., Henneke, G., Flament, D., Querellou, J. and Raffin, J. P. (2009) Binding to PCNA in Euryarchaeal DNA Replication requires two PIP motifs for DNA polymerase D and one PIP motif for DNA polymerase B. J. Mol. Biol. 394, 209-218

28 Tang, X. F., Shen, Y., Matsui, E. and Matsui, I. (2004) Domain topology of the DNA polymerase D complex from a hyperthermophilic archaeon Pyrococcus horikoshii. Biochemistry. 43, 11818-11827

29 Tori, K., Kimizu, M., Ishino, S. and Ishino, Y. (2007) DNA polymerases BI and D from the hyperthermophilic archaeon Pyrococcus furiosus both bind to proliferating cell nuclear antigen with their C-terminal PIP-box motifs. J. Bacteriol. 189, 5652-5657

30 Cann, I. K., Ishino, S., Nomura, N., Sako, Y. and Ishino, Y. (1999) Two family B DNA polymerases from Aeropyrum pernix, an aerobic hyperthermophilic crenarchaeote. J. Bacteriol. 181, 5984-5992

31 Iwai, T., Kurosawa, N., Itoh, Y. H., Kimura, N. and Horiuchi, T. (2000) Sequence analysis of three family B DNA polymerases from the thermoacidophilic crenarchaeon Sulfurisphaera ohwakuensis. DNA Res. 7, 243-251

32 Daimon, K., Kawarabayasi, Y., Kikuchi, H., Sako, Y. and Ishino, Y. (2002) Three proliferating cell nuclear antigen-like proteins found in the hyperthermophilic archaeon Aeropyrum pernix: interactions with the two DNA polymerases. J. Bacteriol. 184, 687-694 
33 Dionne, I., Nookala, R. K., Jackson, S. P., Doherty, A. J. and Bell, S. D. (2003) A heterotrimeric PCNA in the hyperthermophilic archaeon Sulfolobus solfataricus. Mol. Cell. 11, 275-282

34 Dore, A. S., Kilkenny, M. L., Jones, S. A., Oliver, A. W., Roe, S. M., Bell, S. D. and Pearl, L. H. (2006) Structure of an archaeal PCNA1-PCNA2-FEN1 complex: elucidating PCNA subunit and client enzyme specificity. Nucleic Acids Res. 34, 4515-4526

35 Beattie, T. R. and Bell, S. D. (2012) Coordination of multiple enzyme activities by a single PCNA in archaeal Okazaki fragment maturation. EMBO J. 31, 1556-1567

36 Kiyonari, S., Takayama, K., Nishida, H. and Ishino, Y. (2006) Identification of a novel binding motif in Pyrococcus furiosus DNA ligase for the functional interaction with proliferating cell nuclear antigen. J. Biol. Chem. 281, 28023-28032

37 Mayanagi, K., Kiyonari, S., Saito, M., Shirai, T., Ishino, Y. and Morikawa, K. (2009) Mechanism of replication machinery assembly as revealed by the DNA ligase-PCNA-DNA complex architecture. Proc. Natl. Acad. Sci. U.S.A. 106, 4647-4652

38 Erauso, G., Reysenbach, A. L., Godfroy, A., Meunier, J. R., Crump, B., Partensky, F., Baross, J. A., Marteinsson, V., Barbier, G., Pace, N. R. and Prieur, D. (1993) Pyrococcus abyssi sp. nov., a new hyperthermophilic archaeon isolated from a deep-sea hydrothermal vent. Arch. Microbiol. 160, 338-349

39 Cohen, G. N., Barbe, V., Flament, D., Galperin, M., Heilig, R., Lecompte, O., Poch, O., Prieur, D., Querellou, J., Ripp, R., Thierry, J. C., Van der Oost, J., Weissenbach, J., Zivanovic, Y. and Forterre, P. (2003) An integrated analysis of the genome of the hyperthermophilic archaeon Pyrococcus abyssi. Mol. Microbiol. 47, 1495-1512

40 Gueguen, Y., Rolland, J. L., Lecompte, O., Azam, P., Le Romancer, G., Flament, D., Raffin, J. P. and Dietrich, J. (2001) Characterization of two DNA polymerases from the hyperthermophilic euryarchaeon Pyrococcus abyssi. Eur. J. Biochem. 268, 5961-5969

41 Henneke, G., Gueguen, Y., Flament, D., Azam, P., Querellou, J., Dietrich, J., Hübscher, U. and Raffin, J. P. (2002) Replication Factor C from the hyperthermophilic archaeon Pyrococcus abyssi does not need ATP hydrolysis for clamp-loading and contains a functionally conserved RFC PCNA-binding domain. J. Mol. Biol. 323, 795-810

42 Palud, A., Villani, G., L'Haridon, S., Querellou, J., Raffin, J. P. and Henneke, G. (2008) Intrinsic properties of the two replicative DNA polymerases of Pyrococcus abyssi in replicating abasic sites: possible role in DNA damage tolerance? Mol. Microbiol. 70, 746-761

43 Le Laz, S., Le Goaziou, A. and Henneke, G. (2010) Structure-specific nuclease activities of Pyrococcus abyssi RNase HII. J. Bacteriol. 192, 3689-3698

44 Jin, Y. H., Obert, R., Burgers, P. M., Kunkel, T. A., Resnick, M. A. and Gordenin, D. A. (2001) The 3'-->5' exonuclease of DNA polymerase delta can substitute for the 5' flap endonuclease Rad27/Fen1 in processing Okazaki fragments and preventing genome instability. Proc. Natl. Acad. Sci. U.S.A. 98, 5122-5127

45 Mossi, R., Ferrari, E. and Hubscher, U. (1998) DNA ligase I selectively affects DNA synthesis by DNA polymerases delta and epsilon suggesting differential functions in DNA replication and repair. J. Biol. Chem. 273, 14322-14330

46 Creze, C., Ligabue, A., Laurent, S., Lestini, R., Laptenok, S. P., Khun, J., Vos, M. H., Czjzek, M., Myllykallio, H. and Flament, D. (2012) Modulation of the Pyrococcus abyssi NucS endonuclease activity by replication clamp at functional and structural levels. J. Biol. Chem. 287, 15648-15660

47 Zheng, L. and Shen, B. (2011) Okazaki fragment maturation: nucleases take centre stage. J. Mol. Cell. Biol. 3, 23-30 


\section{FIGURE LEGENDS}

Figure 1. PabRNase HII is required to initiate strand displacement DNA synthesis by PabpolB, followed by PabFen1 cleavage. (A) The unlabeled minicircle primer-template (P2:T1) was preincubated at $60^{\circ} \mathrm{C}$ for $10 \mathrm{~min}$ in $\mathrm{PabpolB}$ reaction buffer before addition of dNTPs and PabpolB. Incubation was continued at $60^{\circ} \mathrm{C}$ for 30 min (lanes 4-7). Analysis was on an $8 \%$ nondenaturing PAGE with SYBR Gold present. The structure of the RNA-DNA primer annealed to the minicircle DNA template is depicted at the top. Lane 1, control reaction without protein. Lanes 2 and 3 are the control reactions with enzymes alone, PabpolB and PabRNase HII, respectively. The arrow indicates nicked DNA minicircles (B) The structure of the 5'-FAM-labeled monoribonucleotide DNA primer annealed to the minicircle DNA template (P3:T1) is illustrated at the top. Reactions were run at $60^{\circ} \mathrm{C}$ for 10 min and products analyzed by denaturing PAGE as described under Experimental. PabpolB was incubated with varying amounts of PabFen1 (lanes 3-7). Lanes 2 and 8 are the control reactions with individual enzymes, PabpolB and PabFen1, respectively. Lane 1, control reaction without enzyme. Lane $M$ contains the 5'-FAM labeled 57 nt (C) Delayed addition of PabFen1 to PabpolB strand displacement assays. Reactions used 5'-FAM-labeled monoribonucleotide DNA primer annealed to the minicircle DNA template (P3:T1) and 1 pmol of PabFen1, which was added at the time interval indicated after the start of reaction with PabpolB. Total incubation time is $30 \mathrm{~min}$. Products were electrophoresed and quantified as described in Experimental. Relative distribution of flap cleavage products is expressed as the ratio of different product population sizes (1-12 nt, 12-19 nt and 20-25 nt) / total number of flap cleavage products.

Figure 2. Coordinated activities of PabpolB and PabFen1 on monoribonucleotideprimed DNA replication intermediates produce ligatable nicks. The dual-labeled substrate (P1:P4:T1) is shown at the top. The monoribonucleotide portion is indicated by a thick line, and DNA is indicated by a solid black line. Reactions were performed in the presence of various combinations of PabpolB (0.5 pmol), PabFen1 (0.5 pmol), PabLig1 (2 pmol) and PabPCNA (0.5 pmol) in PabpolB buffer containing ATP $(0.1 \mathrm{mM})$. Upper and lower gels refer to upstream (5'-FAM label) and downstream (3'-Cy5 label) resulting products, respectively. Lane 1 contains oligonucleotide ladders (32-, 57- and 87-nt) 5'-FAM labeled. Plus (+) denotes presence and minus (-) denotes absence of enzyme.

Figure 3. Coordinated activities of PabpolD and PabFen1 on RNA-primed DNA replication intermediates produce ligatable nicks. The dual-labeled substrate (P1:P2:T1) is shown at the top. The RNA portion is indicated by a thick line, and DNA is indicated by a solid black line. Reactions were performed in the presence of various combinations of PabpolD (0.25 pmol), PabFen1 (0.5 pmol) and PabPCNA (0.5 pmol) in PabpolD buffer containing ATP $(0.1 \mathrm{mM})$ under the conditions described in Experimental. Upper and lower gels refer to upstream (5'-FAM label) and downstream (3'-Cy5 label) resulting products, respectively. Lane $M$ contains oligonucleotide markers (32-, 57- and 87-nt) 5'-FAM labeled. Plus (+) denotes presence and minus (-) denotes absence of enzyme.

Figure 4. Initial cleavage event by PabRNase HII facilitates RNA primer removal of Okazaki fragments. The dual-labeled substrate (P1:P2:T1) is shown at the top. Initial cleavage events were performed by incubating PabRNase HII $(1 \mathrm{pmol})$ at $60^{\circ} \mathrm{C}$ for $10 \mathrm{~min}$. Subsequent reactions were carried out at $60^{\circ} \mathrm{C}$ for $30 \mathrm{~min}$ in the presence of various combinations of either PabpolD (0.25 pmol) (lanes 2-9) or PabpolB (0.5 pmol) (lanes 11-18), PabFen1 (0.5 pmol), PabPCNA (0.5 pmol) and PabLig1 (2 pmol) in the respective 
polymerase buffer containing ATP $(0.1 \mathrm{mM})$ under the conditions described in Experimental. Upper and lower gels refer to the upstream (5'-FAM label) and downstream (3'-Cy5 label) resulting products, respectively. $M$ corresponds to oligonucleotide ladders (32-, 57- and 87-nt) 5'-FAM labeled. Plus (+) denotes presence and minus (-) denotes absence of enzyme.

Figure 5. Model for RNA primer removal in archaea. Complete RNA primer elimination is ensured by continuous pol D strand displacement DNA synthesis and 5'-RNA flap cleavage by Fen1 (steps 1 and 2). These coupled activities are iterated until all RNA is degraded. Then, DNA ligase 1 is recruited to seal the resulting nick, generating the continuous double-stranded DNA (step 3). Alternatively, the processing maturation requires a site-specific cleavage by RNase HII of the RNA initiator (step 1'). Removal of the last 5' ribonucleotide is accomplished by Fen1 cleavage and strand displacement DNA synthesis by pol B or pol D (steps 2' and 3'). A nick is then generated between the two DNA termini (step 3') which are joined by DNA ligase 1 (step 4'). 
FIGURE 1

A

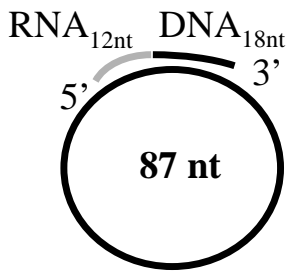

PabpolB (pmol) $\quad 0 \quad 2 \quad 2 \quad 0 \quad 0.25 \quad 0.5 \quad 1 \quad 2$ 1 pmol PabRNaseHII - $\quad+\quad+\quad+++$

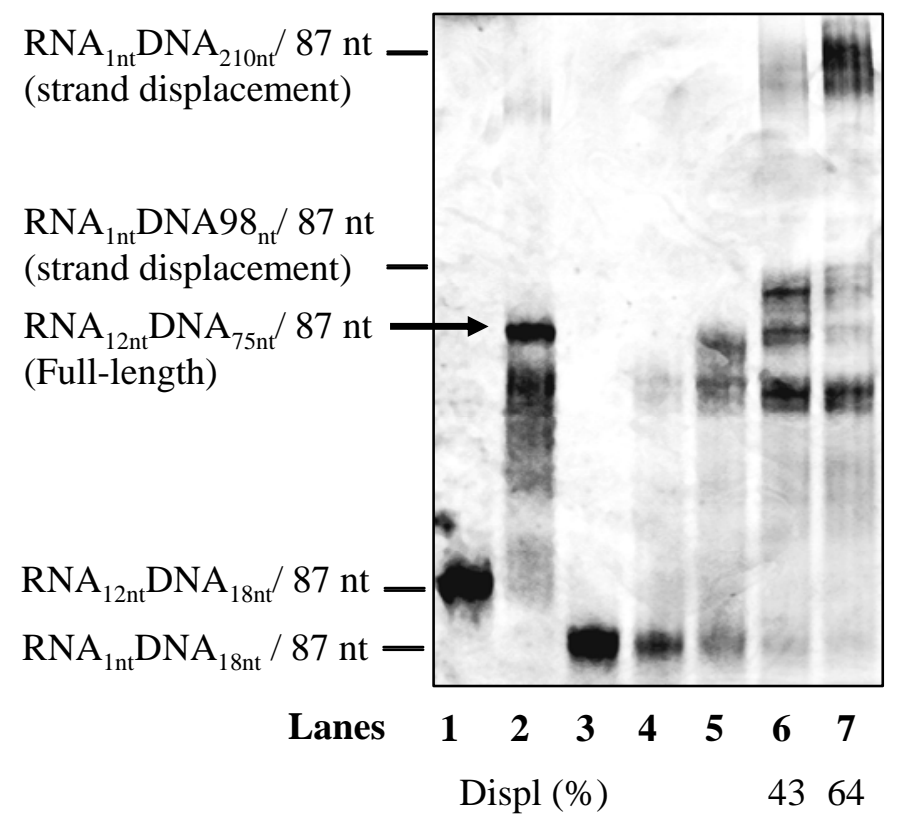

B

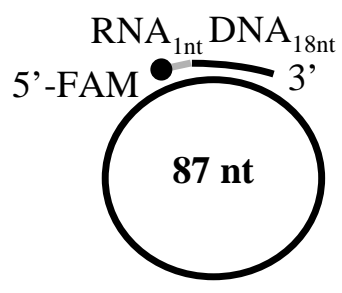

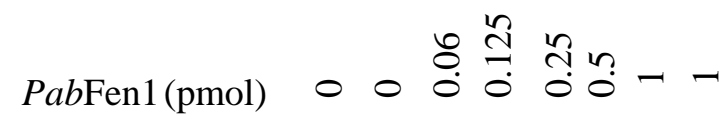
0.5 pmol PabpolB - ++++++

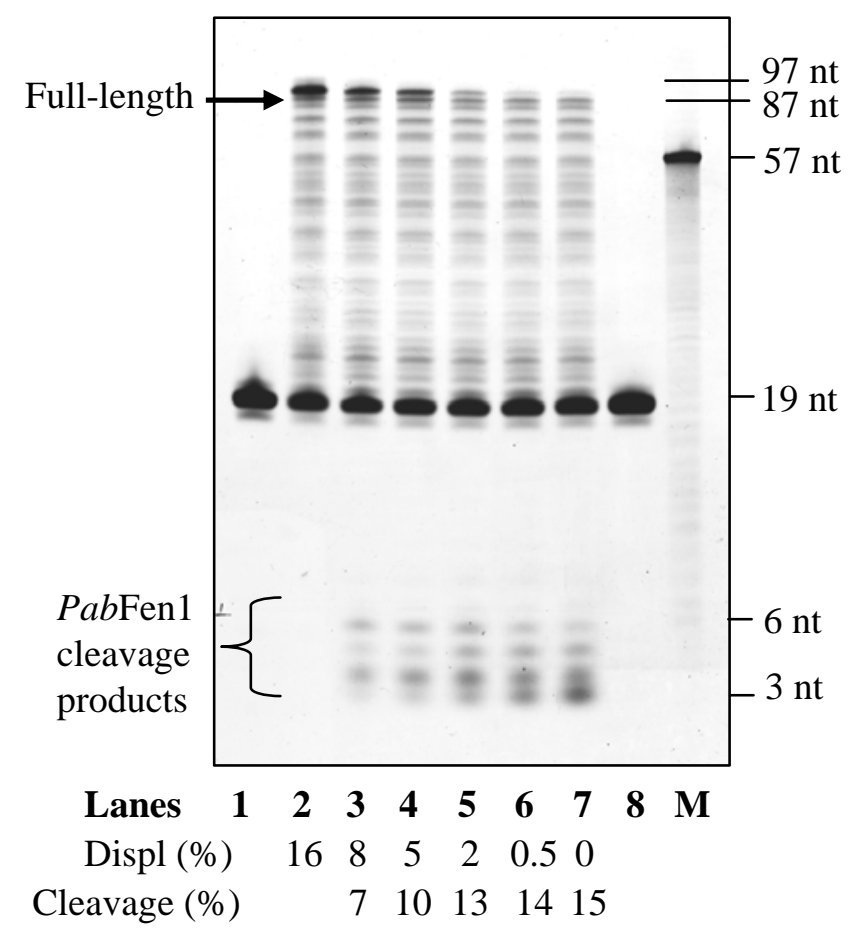

C

Relative amount of PabFen1 cleavage products (\%) 100

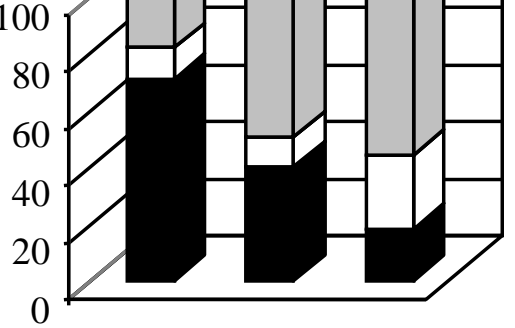

20-25

12-19 Size of flap cleavage

1-12 products (nt)

Time of Fen1 addition (min) $\quad \begin{array}{lll}0 & 5 & 15\end{array}$ 
FIGURE 2
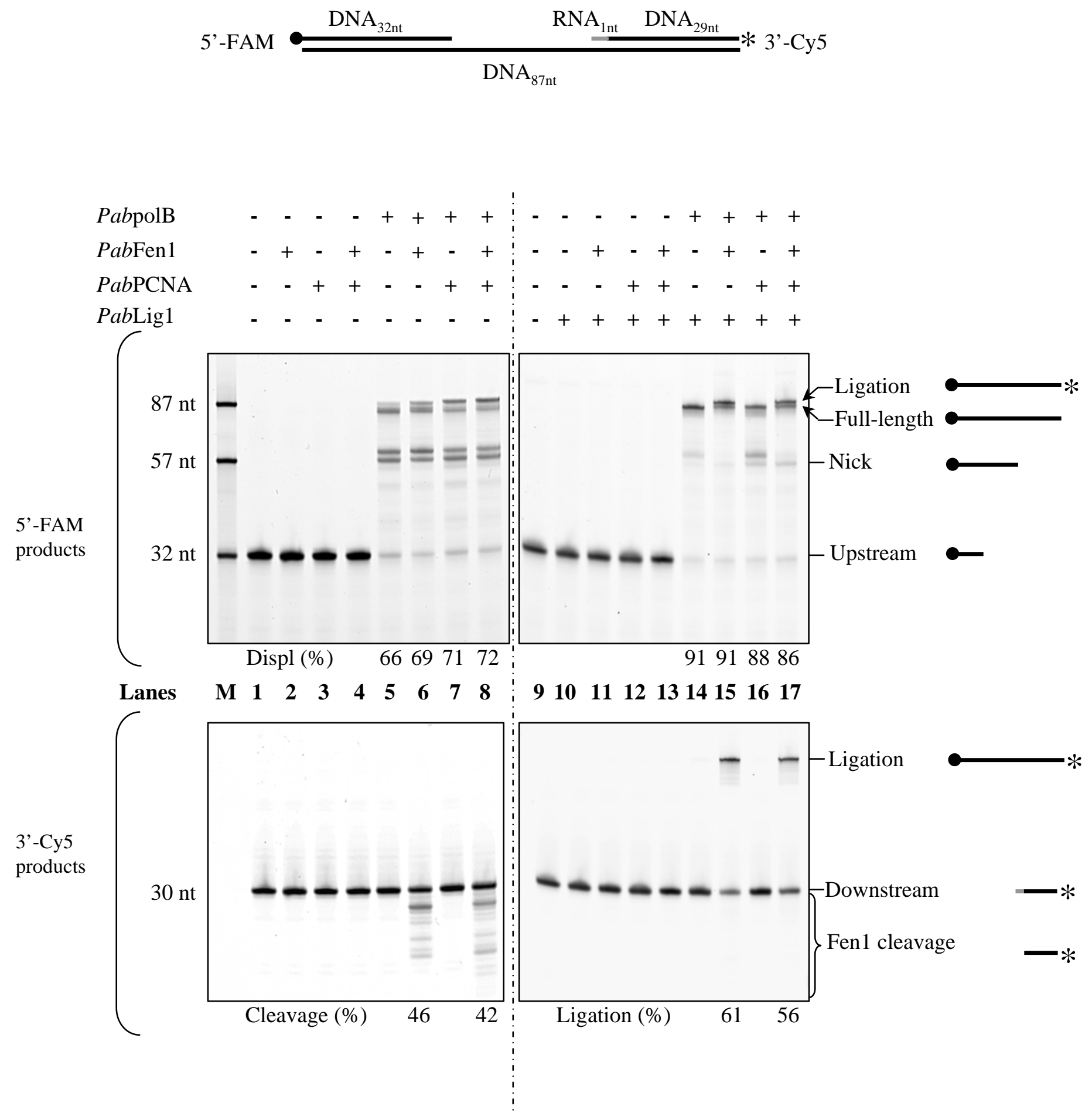
FIGURE 3
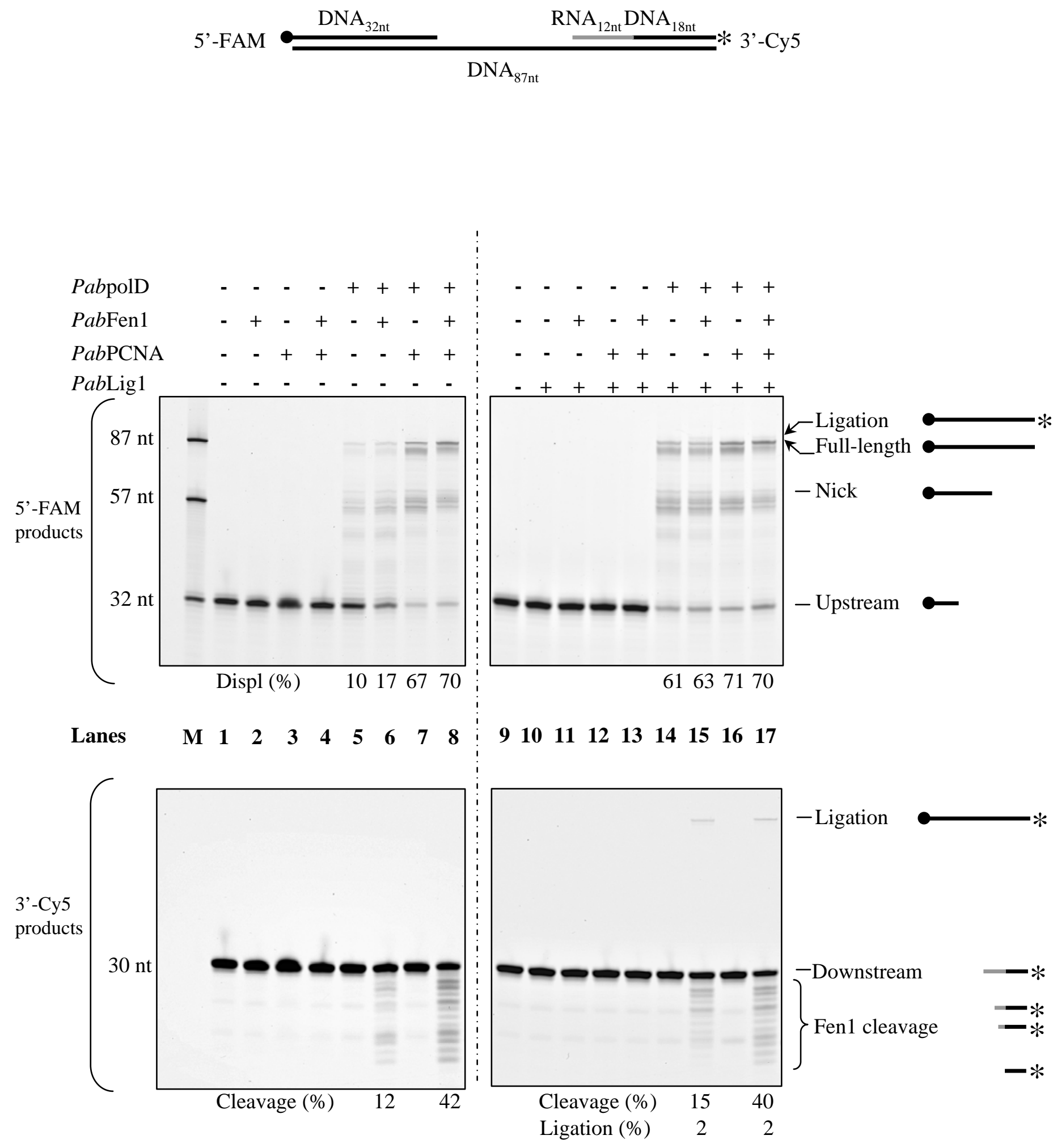
FIGURE 4

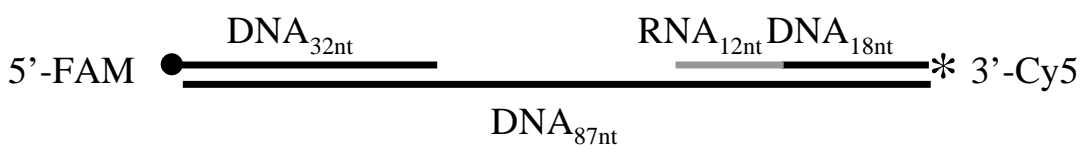

PabpolB

PabpolD

PabFen1

PabPCNA

PabLig1

PabRNase HII
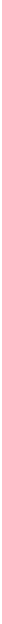

$\begin{array}{lllllllllll}\text { Lanes } & M & 1 & 2 & 3 & 4 & 5 & 6 & 7 & 8 & 9\end{array}$

$\begin{array}{lllllllll}10 & 11 & 12 & 13 & 14 & 15 & 16 & 17 & 18\end{array}$
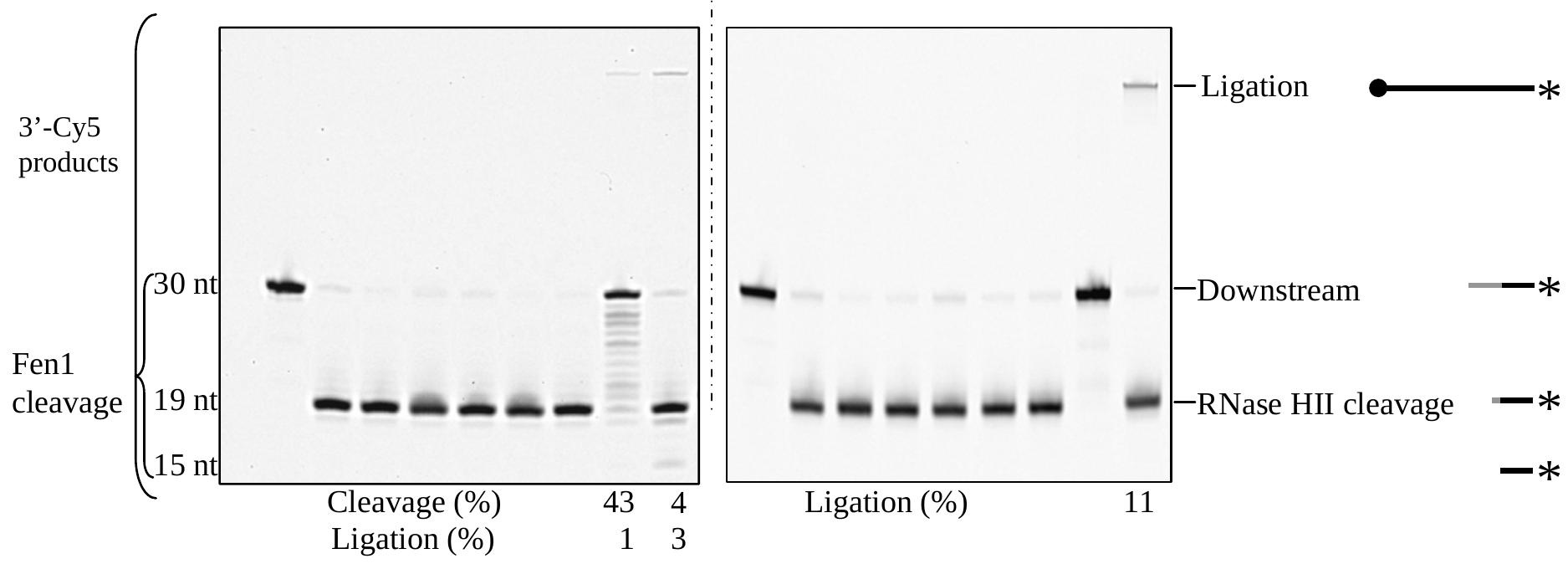
FIGURE 5

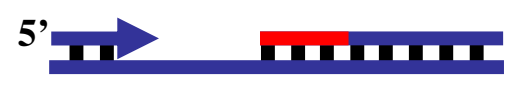

(1')
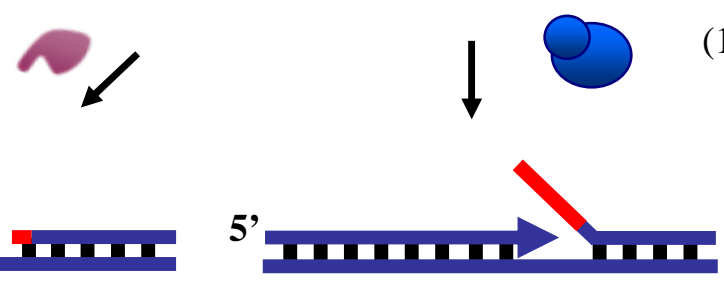

(2')

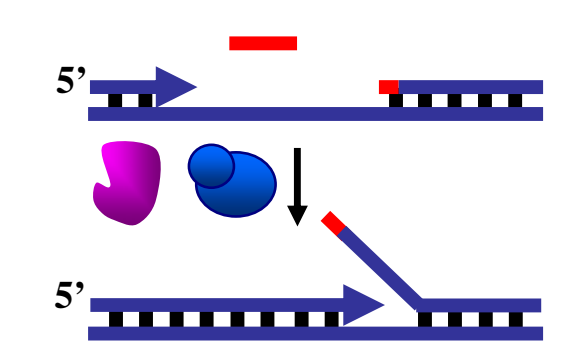

(3')

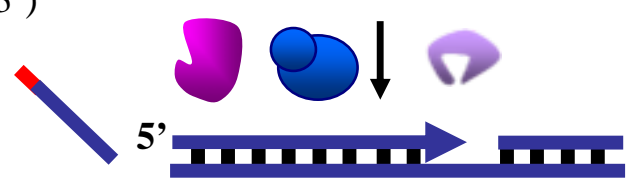

(4') \}
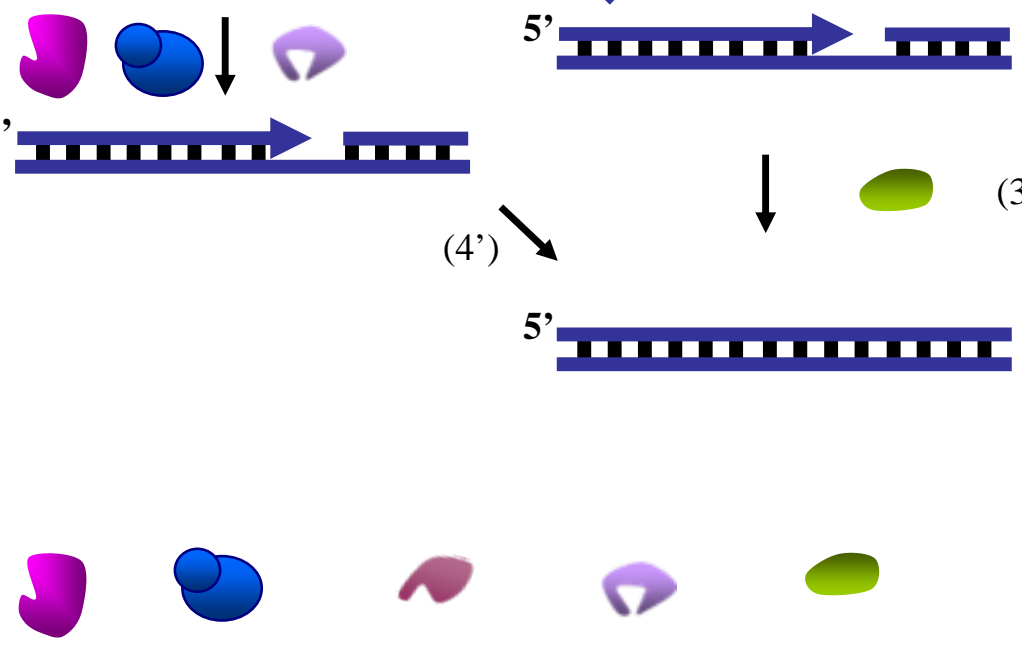

pol B

$$
\text { pol D }
$$

RNase HII
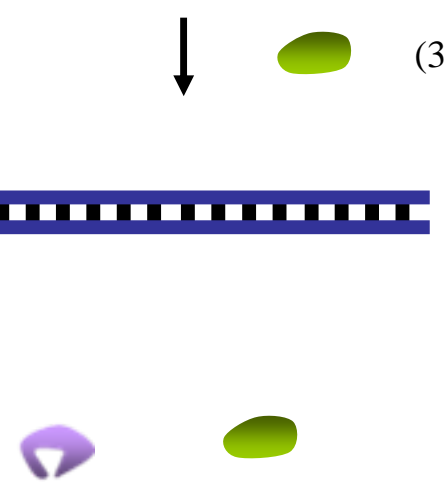

(1) 